\title{
An Efficient and Stable Spectral-Element Method for Acoustic Scattering by an Obstacle
}

\author{
Jing $\mathrm{An}^{1}$ and Jie Shen ${ }^{1,2, *}$ \\ ${ }^{1}$ School of Mathematics and Science, Xiamen University, Xiamen 361005, China. \\ 2 Department of Mathematics, Purdue University, West Lafayette, IN 47907, USA. \\ Received 6 July 2013; Accepted (in revised version) 8 August 2013
}

Available online 31 August 2013

\begin{abstract}
A spectral-element method is developed to solve the scattering problem for time-harmonic sound waves due to an obstacle in an homogeneous compressible fluid. The method is based on a boundary perturbation technique coupled with an efficient spectral-element solver. Extensive numerical results are presented, in order to show the accuracy and stability of the method.
\end{abstract}

AMS subject classifications: 78A45, 65N35, 35J05, 41A58

Key words: Spectral method, acoustic scattering, boundary perturbation, Helmholtz equation.

\section{Introduction}

The scattering of acoustic and electromagnetic waves is important in a wide range of problems of scientific and technological interest, and various numerical techniques have been proposed to solve related problems - cf. the book [3] and two surveys [16, 23] for example. A particularly compelling class of methods is based on boundary perturbations [2], and can be traced back to the work of Rayleigh [15] and Rice [17]. However, such algorithms depend upon significant cancellations for convergence, so their numerical implementations are highly ill-conditioned when pursued to high order $[11,12]$. A new boundary perturbation algorithm called the transformed field expansion (TFE) that does not rely on strong cancellations for convergence was therefore proposed, and proved to be not only accurate and stable but also robust at high order [11-13].

The main purpose of this article is to apply the well developed method in Ref. [13] to the scattering problem for time-harmonic acoustic waves due to an obstacle in an homogeneous compressible fluid. In particular, we construct a well-conditioned spectral-element solver for the Helmholtz equation, which repeatedly involves the TFE algorithm. This solver is highly efficient and accurate because it exploits the fact that the Dirichlet-toNeumann operator (DNO) is global in physical space but local in frequency space, hence

*Corresponding author. Email addresses: aj154@163.com (J. An), shen7@purdue.edu (J. Shen) 
we can reduce the two-dimensional problem to a sequence of one-dimensional problems using a spectrally accurate approximation to the DNO. We demonstrate that this provides an efficient numerical solution, as the frequency of the scattered radiation is varied from low to moderate.

In Section 2 we formulate the governing equations for an electromagnetic field incident upon a periodic two-dimensional irregular grating. In Section 3, we present the Method of Transformed Field Expansions, where we make a change of variables followed by an expansion in terms of the boundary perturbation. We then construct our two-domain spectral-element method to solve the resulting two-point boundary value problem in Section 4 and present the consequent numerical results in Section 5, followed by concluding remarks in Section 6. Finally, some of the detailed formulae needed in Section 3 are summarised in the Appendix.

\section{Governing Equations}

Given an incident field $\phi$ on a bounded obstacle $\Omega^{+} \subset \mathbb{R}^{2}$ in the homogeneous compressible fluid, the scattered field in the exterior of the obstacle, $\Omega^{-}=\mathbb{R}^{2} \backslash \bar{\Omega}^{+}$can be written as

$$
u^{-}=P_{e}-\phi
$$

where $P_{e}$ is the (total) acoustic pressure. The governing equation for $u^{-}$is

$$
\Delta u^{-}+\left(k^{-}\right)^{2} u^{-}=0 \text { in } \Omega^{-},
$$

where $k^{-}=\omega / c^{-}$is the wave number (assumed to be real and positive), $\omega$ is the frequency, and $c^{-}$is the sound speed in $\Omega^{-}$. We assume that the incident field $\phi$ satisfies (2.1) everywhere, except possibly at some places in $\Omega^{-}$such that $\phi$ could correspond to a point source in $\Omega^{-}$for example. We require that $u^{-}$satisfies the Sommerfeld radiation condition at infinity. Within the obstacle $\Omega^{+}$, the reflected acoustic pressure $u^{+}$satisfies the Bergmann equation $[1,6]$

$$
\Delta u^{+}+\left(k^{+}\right)^{2} u^{+}=0, \quad \text { in } \Omega^{+},
$$

where $k^{+}=\omega / c^{+}$with $c^{+}$denoting the sound speed in $\Omega^{+}$. At the interface $S=\partial \Omega^{+}$, we have a pair of transmission conditions expressing continuity of pressure and normal velocity - viz.

$$
P_{e}=u^{+} \text {and } \frac{1}{\rho_{-}} \frac{\partial P_{e}}{\partial n}=\frac{1}{\rho_{+}} \frac{\partial u^{+}}{\partial n} \text { on } S
$$


where $\rho_{+}, \rho_{-}$denote the constant densities of the fluid in $\Omega^{+}, \Omega^{-}$, respectively. In summary, we seek to solve the following problem:

$$
\begin{aligned}
& \Delta u^{+}+\left(k^{+}\right)^{2} u^{+}=0 \text { in } \Omega^{+}, \\
& \Delta u^{-}+\left(k^{-}\right)^{2} u^{-}=0 \text { in } \Omega^{-}, \\
& u^{+}-u^{-}=\phi \text { on } S, \\
& \frac{1}{\rho_{+}} \frac{\partial u^{+}}{\partial n}-\frac{1}{\rho_{-}} \frac{\partial u^{-}}{\partial n}=\frac{1}{\rho_{-}} \frac{\partial \phi}{\partial n} \text { on } S, \\
& \lim _{r \rightarrow \infty} r^{\frac{1}{2}}\left(\partial_{r} u^{-}-\mathrm{i} k^{-} u^{-}\right)=0,
\end{aligned}
$$

in which Eq. (2.7) can be re-stated as

$$
\frac{\partial u^{+}}{\partial n}-\delta \frac{\partial u^{-}}{\partial n}=\delta \frac{\partial \phi}{\partial n} \text { on } S, \text { where } \delta=\rho_{+} / \rho_{-} .
$$

We now assume that the obstacle is a star-shaped domain — i.e. there exists $g(\theta)$ such that

$$
\Omega^{+}=\{(r, \theta): 0 \leq r<a+g(\theta), 0 \leq \theta<2 \pi\}
$$

and we also write

$$
\begin{aligned}
& \Omega^{-}=\{(r, \theta): r>a+g(\theta), 0 \leq \theta<2 \pi\}, \\
& S=\{(r, \theta): r=a+g(\theta), 0 \leq \theta<2 \pi\} .
\end{aligned}
$$

Let $b$ be such that $b>a+|g|_{L^{\infty}}$. Then using the classical argument of separation of variables, the general solution of Eqs. (2.5) and (2.8) for $r \geq b$ can be expressed as

$$
u^{-}(r, \theta)=\sum_{p=-\infty}^{\infty} a_{p} H_{p}^{(1)}\left(k^{-} r\right) e^{\mathrm{i} p \theta}
$$

where $H_{p}^{(1)}$ is the $p$-th degree Hankel function of the first kind. Hence from the expression

$$
u^{-}(b, \theta)=\psi(\theta)=\sum_{p=-\infty}^{\infty} \hat{\psi}_{p} e^{\mathrm{i} p \theta},
$$

the coefficients $a_{p}$ in Eq. (2.10) can be determined uniquely such that

$$
u^{-}(r, \theta)=\sum_{p=-\infty}^{\infty} \hat{\psi}_{p} \frac{H_{p}^{(1)}\left(k^{-} r\right)}{H_{p}^{(1)}\left(k^{-} b\right)} e^{\mathrm{i} p \theta}
$$


We define an operator $T$ by

$$
T \psi=\partial_{r} u^{-}(b, \theta)=\sum_{p=-\infty}^{\infty} k^{-} \frac{d_{z} H_{p}^{(1)}\left(k^{-} b\right)}{H_{p}^{(1)}\left(k^{-} b\right)} \hat{\psi}_{p} e^{\mathrm{i} p \theta},
$$

where $d_{z} H_{p}^{(1)}\left(k^{-} b\right)=d H_{p}^{(1)}(z) /\left.d z\right|_{z=k^{-} b}$. This operator $T$ maps the Dirichlet data $\psi$ to Neumann data $\left.\partial_{r} u^{-}\right|_{r=b}$, so it is commonly referred as the Dirichlet-to-Neumann operator (DNO).

Let us denote $\Omega_{b}^{-}=\Omega^{-} \cap\{(r, \theta): r<b\}$. Using the operator $T$ and bearing in mind Eq. (2.9), we can re-state Eqs. (2.4)-(2.8) as

$$
\begin{aligned}
& \Delta u^{+}+\left(k^{+}\right)^{2} u^{+}=0 \text { in } \Omega^{+}, \\
& \Delta u^{-}+\left(k^{-}\right)^{2} u^{-}=0 \text { in } \Omega_{b}^{-}, \\
& u^{+}-u^{-}=\phi \text { on } S, \\
& \frac{\partial u^{+}}{\partial n}-\delta \frac{\partial u^{-}}{\partial n}=\delta \frac{\partial \phi}{\partial n} \text { on } S, \\
& \partial_{r} u^{-}(b, \theta)-T u^{-}(b, \theta)=0 .
\end{aligned}
$$

\section{Transformed Field Expansion}

Although the problem (2.12)-(2.16) is linear with constant coefficients, it involves a general interface $S$ that prevents us from developing fast solvers through the classical technique of separation of variables. This difficulty can be circumvented by using a boundary perturbation method [2]. However, a direct application of the boundary perturbation method can lead to very ill-conditioned recursions [9], and it has been shown that the transformed field expansion (TFE) method may dramatically improve the conditioning of the resulting recursions $[8,10]$.

The TFE method consists of two essential steps: (1) "domain flattening" through a simple change of variables; and (2) boundary perturbation. The TFE method for acoustic scattering by an homogeneous obstacle was developed in Ref. [13], and we now extend that algorithm to solve our present problem.

\subsection{Change of variables}

We introduce the following change of variables:

$$
\begin{aligned}
\theta^{\prime} & =\theta, \\
r^{\prime} & =\frac{a r}{a+g}, \quad r<a+g, \\
r^{\prime} & =\frac{d r-b g}{d-g}, \quad a+g<r<b,
\end{aligned}
$$


where $d=b-a$. One can easily check that the domain $\Omega^{+}=\{(r, \theta): r<a+g(\theta)\}$ is transformed into the disk $\Omega_{a}=\left\{\left(r^{\prime}, \theta^{\prime}\right): r^{\prime}<a\right\}$, and we have the following differentiation rules:

$$
\left\{\begin{array}{lll}
(a+g) \partial_{\theta} & =(a+g) \partial_{\theta^{\prime}}-r^{\prime} \partial_{\theta^{\prime}} g \partial_{r^{\prime}}, & \\
(a+g) \partial_{r} & =a \partial_{r^{\prime}}, & \text { for } r<a+g(\theta) \\
(a+g) D_{r} & =(a+g) D_{r^{\prime}}, &
\end{array}\right.
$$

and

$$
\left\{\begin{array}{ll}
(d-g) \partial_{\theta}=(d-g) \partial_{\theta^{\prime}}-B\left(r^{\prime}, \theta^{\prime}\right) \partial_{r^{\prime}}, \\
(d-g) \partial_{r}=d \partial_{r^{\prime}} \\
(d-g) D_{r}=d D_{r^{\prime}}+A\left(r^{\prime}, \theta^{\prime}\right) \partial_{r^{\prime}},
\end{array} \quad \text { for } a+g(\theta)<r<b\right.
$$

where

$$
\begin{aligned}
& D_{r}=r \partial_{r}, \quad A\left(r^{\prime}, \theta^{\prime}\right)=g(\theta)\left(b-r^{\prime}\right), \\
& B\left(r^{\prime}, \theta^{\prime}\right)=\partial_{\theta^{\prime}} A=\left(\partial_{\theta^{\prime}} g\left(\theta^{\prime}\right)\right)\left(b-r^{\prime}\right) .
\end{aligned}
$$

We note also that $d r=d r^{\prime}+A\left(r^{\prime}, \theta^{\prime}\right)$.

Let us write $\Omega_{a b}=\left\{\left(r^{\prime}, \theta^{\prime}\right): a<r^{\prime}<b\right\}$ and $\tilde{u}^{ \pm}\left(r^{\prime}, \theta^{\prime}\right)=u^{ \pm}(r, \theta), \tilde{\phi}\left(r^{\prime}, \theta^{\prime}\right)=\phi(r, \theta)$. After some tedious but straightforward manipulation (cf. the Appendix), we transform the system (2.12)-(2.16) to the new coordinates $\left(r^{\prime}, \theta^{\prime}\right)$ :

$$
\begin{aligned}
& D_{r^{\prime}}^{2} \tilde{u}^{+}+\partial_{\theta^{\prime}}^{2} \tilde{u}^{+}+\left(r^{\prime}\right)^{2}\left(k^{+}\right)^{2} \tilde{u}^{+}=F_{1}\left(r^{\prime}, \theta^{\prime} ; \tilde{u}^{+}\right) \text {in } \Omega_{a} \\
& D_{r^{\prime}}^{2} \tilde{u}^{-}+\partial_{\theta^{\prime}}^{2} \tilde{u}^{-}+\left(r^{\prime}\right)^{2}\left(k^{-}\right)^{2} \tilde{u}^{-}=F_{2}\left(r^{\prime}, \theta^{\prime} ; \tilde{u}^{-}\right) \text {in } \Omega_{a b} \\
& \tilde{u}^{+}\left(a, \theta^{\prime}\right)-\tilde{u}^{-}\left(a, \theta^{\prime}\right)=\tilde{\phi}\left(a, \theta^{\prime}\right) \\
& \left(L_{1} \frac{\partial \tilde{u}^{+}}{\partial r^{\prime}}-L_{2} \frac{\partial \tilde{u}^{+}}{\partial \theta^{\prime}}\right)-\delta\left(L_{3} \frac{\partial \tilde{u}^{-}}{\partial r^{\prime}}-L_{2} \frac{\partial \tilde{u}^{-}}{\partial \theta^{\prime}}\right)=\delta\left(L_{3} \frac{\partial \tilde{\phi}}{\partial r^{\prime}}-L_{2} \frac{\partial \tilde{\phi}}{\partial \theta^{\prime}}\right) \text { at } r^{\prime}=a, \\
& \partial_{r^{\prime}} \tilde{u}^{-}\left(b, \theta^{\prime}\right)-T \tilde{u}^{-}\left(b, \theta^{\prime}\right)=J\left(\theta^{\prime} ; \tilde{u}^{-}\right)
\end{aligned}
$$

where $F_{1}$ and $F_{2}$ have the explicit expressions given in (A.1)-(A.2) that contain all of the terms involving the function $g(\theta)$, and

$$
\begin{aligned}
& L_{1}=\left(a+\frac{a\left(\partial_{\theta^{\prime}} g\right)^{2}}{(a+g)^{2}}\right), \quad L_{2}=\frac{\partial_{\theta^{\prime}} g}{a+g}, \quad L_{3}=\frac{d(a+g)}{d-g}-\frac{(a-b)\left(\partial_{\theta^{\prime}} g\right)^{2}}{(d-g)(a+g)}, \\
& d J\left(\theta^{\prime} ; \tilde{u}^{-}\right)=-g\left(\theta^{\prime}\right) T \tilde{u}^{-}\left(b, \theta^{\prime}\right) .
\end{aligned}
$$

A graphical sketch of this procedure is depicted in Fig. 1. 


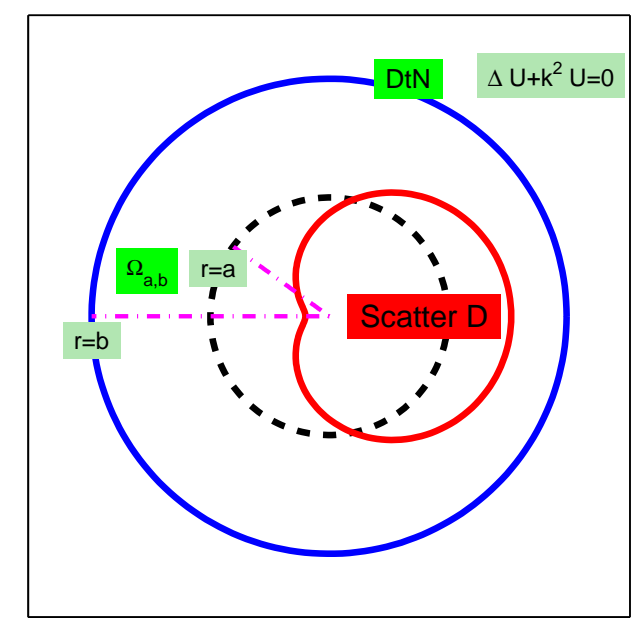

Figure 1: A schematic sketch of the domain flattening and reduction.

\subsection{Recursion by boundary perturbation}

We now describe a boundary perturbation algorithm to solve the transformed system (3.2)-(3.6). Writing $g=\varepsilon f$ and assuming that $f$ is sufficiently smooth, one can show that the transformed fields $\tilde{u}^{ \pm}$are analytic with respect to $\varepsilon-\mathrm{cf}$. $\left.[2,7,11,12]\right)$. Consequently, we can introduce the Taylor expansion

$$
\tilde{u}^{ \pm}\left(r^{\prime}, \theta^{\prime} ; \varepsilon\right)=\sum_{n=0}^{\infty} \tilde{u}_{n}^{ \pm}\left(r^{\prime}, \theta^{\prime}\right) \varepsilon^{n}
$$

Given the incident field

$$
\phi(r, \theta)=e^{\mathrm{i} r(\alpha \cos \theta-\beta \sin \theta)},
$$

for $a+g \leq r \leq b$ we have

$$
\tilde{\phi}\left(r^{\prime}, \theta^{\prime}\right)=e^{\mathrm{i}\left(\frac{(d-g) r^{\prime}+b g}{d}\right)\left(\alpha \cos \theta^{\prime}-\beta \sin \theta^{\prime}\right)}=e^{\mathrm{i} p \theta^{\prime} \frac{\left(b-r^{\prime}\right) f}{d} \varepsilon} e^{\mathrm{i} p \theta^{\prime} r^{\prime}},
$$

so in particular

$$
\tilde{\phi}\left(a, \theta^{\prime}\right)=e^{\mathrm{i} p \theta^{\prime} f \varepsilon} e^{\mathrm{i} a p \theta^{\prime}}=\sum_{n=0}^{\infty} e^{\mathrm{i} a p \theta^{\prime}} \frac{\left(\mathrm{i} p \theta^{\prime} f\right)^{n}}{n !} \varepsilon^{n}:=\sum_{n=0}^{\infty} \tilde{\phi}_{n} \varepsilon^{n} .
$$


Inserting Eqs. (3.7) and (3.9) into Eqs. (3.2)-(3.6) and collecting all terms in $\varepsilon^{n}$, after a straightforward albeit tedious calculation we obtain the following recursions for $\tilde{u}_{n}^{ \pm}$:

$$
\begin{aligned}
& D_{r^{\prime}}^{2} \tilde{u}_{n}^{+}+\partial_{\theta^{\prime}}^{2} \tilde{u}_{n}^{+}+\left(r^{\prime}\right)^{2}\left(k^{+}\right)^{2} \tilde{u}_{n}^{+}=F_{1 n} \text { in } \Omega_{a}, \\
& D_{r^{\prime}}^{2} \tilde{u}_{n}^{-}+\partial_{\theta^{\prime}}^{2} \tilde{u}_{n}^{-}+\left(r^{\prime}\right)^{2}\left(k^{-}\right)^{2} \tilde{u}_{n}^{-}=F_{2 n} \text { in } \Omega_{a b}, \\
& \tilde{u}_{n}^{+}\left(a, \theta^{\prime}\right)-\tilde{u}_{n}^{-}\left(a, \theta^{\prime}\right)=\tilde{\phi}_{n}\left(a, \theta^{\prime}\right), \\
& \partial_{r^{\prime}} \tilde{u}_{n}^{+}-\delta \partial_{r^{\prime}} \tilde{u}_{n}^{-}=Q_{n} \text { on } r^{\prime}=a, \\
& \partial_{r^{\prime}} \tilde{u}_{n}^{-}\left(b, \theta^{\prime}\right)-T \tilde{u}_{n}^{-}\left(b, \theta^{\prime}\right)=J_{n},
\end{aligned}
$$

where the exact expressions for $F_{1 n}, F_{2 n}, Q_{n}$ and $J_{n}$ are given in the Appendix - -cf. Eqs. (A.3)-(A.6).

Eqs. (3.10) and (3.11) are now set in a separable domain where the interface is the circle $r^{\prime}=a$, so we can apply the classical technique of separation of variables. We first expand all the quantities in Fourier series in $\theta^{\prime}$ - i.e. we write

$$
\begin{array}{ll}
\tilde{u}_{n}^{ \pm}\left(r^{\prime}, \theta^{\prime}\right)=\sum_{\tilde{p}=-\infty}^{\infty} \tilde{u}_{n, \tilde{p}}^{ \pm}\left(r^{\prime}\right) e^{\mathrm{i} \tilde{p} \theta^{\prime}}, & F_{1 n}\left(r^{\prime}, \theta^{\prime}\right)=\sum_{\tilde{p}=-\infty}^{\infty} F_{1 n, \tilde{p}}\left(r^{\prime}\right) e^{\mathrm{i} \tilde{p} \theta^{\prime}} \\
F_{2 n}\left(r^{\prime}, \theta^{\prime}\right)=\sum_{\tilde{p}=-\infty}^{\infty} F_{2 n, \tilde{p}}\left(r^{\prime}\right) e^{\mathrm{i} \tilde{p} \theta^{\prime}}, & \tilde{\phi}_{n}\left(a, \theta^{\prime}\right)=\sum_{\tilde{p}=-\infty}^{\infty} \tilde{\phi}_{n, \tilde{p}} e^{\mathrm{i} \tilde{p} \theta^{\prime}}, \\
Q_{n}\left(a, \theta^{\prime}\right)=\sum_{\tilde{p}=-\infty}^{\infty} Q_{n, \tilde{p}} e^{\mathrm{i} \tilde{p} \theta^{\prime}}, & J_{n}\left(b, \theta^{\prime}\right)=\sum_{\tilde{p}=-\infty}^{\infty} J_{n, \tilde{p}} e^{\mathrm{i} \tilde{p} \theta^{\prime}} .
\end{array}
$$

On inserting these Fourier expansions into Eqs. (3.10)-(3.14) and taking Eq. (2.11) into account, we obtain a sequence of one-dimensional equations for $\tilde{u}_{n, \tilde{p}}^{ \pm}$:

$$
\begin{aligned}
& D_{r^{\prime}}^{2} \tilde{u}_{n, \tilde{p}}^{+}-\tilde{p}^{2} \tilde{u}_{n, \tilde{p}}^{+}+\left(r^{\prime}\right)^{2}\left(k^{+}\right)^{2} \tilde{u}_{n, \tilde{p}}^{+}=F_{1 n, \tilde{p}}, \quad 0<r^{\prime}<a, \\
& D_{r^{\prime}}^{2} \tilde{u}_{n, \tilde{p}}^{-}-\tilde{p}^{2} \tilde{u}_{n, \tilde{p}}^{-}+\left(r^{\prime}\right)^{2}\left(k^{-}\right)^{2} \tilde{u}_{n, \tilde{p}}^{-}=F_{2 n, \tilde{p}}, \quad a<r^{\prime}<b, \\
& \tilde{u}_{n, \tilde{p}}^{+}(a)-\tilde{u}_{n, \tilde{p}}^{-}(a)=\tilde{\phi}_{n, \tilde{p}} \text {, } \\
& \partial_{r^{\prime}} \tilde{u}_{n, \tilde{p}}^{+}(a)-\delta \partial_{r^{\prime}} \tilde{u}_{n, \tilde{p}}^{-}(a)=Q_{n, \tilde{p}}, \\
& \partial_{r^{\prime}} \tilde{u}_{n, \tilde{p}}^{-}(b)-k^{-} \frac{d_{z} H_{\tilde{p}}^{(1)}\left(k^{-} b\right)}{H_{\tilde{p}}^{(1)}\left(k^{-} b\right)} \tilde{u}_{n, \tilde{p}}^{-}(b)=J_{n, \tilde{p}} .
\end{aligned}
$$

The above system should be supplemented with the essential pole condition [20]

$$
\tilde{u}_{n \tilde{p}}^{+}(0)=0, \quad \text { for } \tilde{p} \neq 0 .
$$


In summary, we can express the solutions $\tilde{u}^{ \pm}$of Eqs. (3.2)-(3.6) as

$$
\tilde{u}^{ \pm}\left(r^{\prime}, \theta^{\prime}\right)=\sum_{n=0}^{\infty} \sum_{\tilde{p}=-\infty}^{\infty} \tilde{u}_{n, \tilde{p}}^{ \pm}\left(r^{\prime}\right) e^{i \tilde{p} \theta^{\prime}} \varepsilon^{n}
$$

such that our main computational task is to obtain the set $\left\{\tilde{u}_{n, \tilde{p}}^{ \pm}\right\}$as the solutions of Eqs. (3.15)-(3.19) for all $n$ and $\tilde{p}$.

\section{A Two-Domain Spectral-Element Method}

After dropping the subscript $p$ and the superscripted tilde from Eqs. (3.15)-(3.19), It emerges that we have the following generic one-dimensional problem for each pair of $n$ and $p$ :

$$
\begin{aligned}
& L_{p}^{+} u^{+}(r):=D_{r}^{2} u^{+}(r)-p^{2} u^{+}(r)+r^{2}\left(k^{+}\right)^{2} u^{+}(r)=F_{1}(r), \quad 0<r<a, \\
& L_{p}^{-} u^{-}(r):=D_{r}^{2} u^{-}(r)-p^{2} u^{-}(r)+r^{2}\left(k^{-}\right)^{2} u^{-}(r)=F_{2}(r), \quad a<r<b, \\
& u^{+}(a)-u^{-}(a)=\phi, \quad \partial_{r} u^{+}(a)-\delta \partial_{r} u^{-}(a)=Q, \\
& u^{+}(0)=0 \text { for } p \neq 0, \text { and } \partial_{r} u^{-}(b)-k^{-} \frac{d_{z} H_{p}^{(1)}\left(k^{-} b\right)}{H_{p}^{(1)}\left(k^{-} b\right)} u^{-}(b)=J .
\end{aligned}
$$

We describe below a two-domain spectral-element method for solving this system.

\subsection{Weak formulation}

We first homogenise the interface and boundary conditions in Eqs. (4.1)-(4.4) and rewrite the system in a suitable form for a Galerkin approximation. Thus denoting

$$
T_{p}=k^{-} \frac{d_{z} H_{p}^{(1)}\left(k^{-} b\right)}{H_{p}^{(1)}\left(k^{-} b\right)}
$$

and setting

$$
\begin{aligned}
& u^{*+}=\left(\frac{\delta\left(T_{p}(\phi-Q a)-J\right)}{T_{p} a(\delta-1)-\left(1-T_{p} b\right)}+Q\right) r, \\
& u^{*-}=\frac{T_{p}(\phi-Q a)-J}{T_{p} a(\delta-1)-\left(1-T_{p} b\right)} r+a(\delta-1) \frac{T_{p}(\phi-Q a)-J}{T_{p} a(\delta-1)-\left(1-T_{p} b\right)}-\phi+Q a, \\
& \hat{u}^{+}=u^{+}-u^{*+}, \quad \hat{u}^{-}=u^{-}-u^{*-},
\end{aligned}
$$


we can rewrite Eqs. (4.1)-(4.4) as

$$
\begin{aligned}
& D_{r}\left(\frac{1}{\rho_{+}} D_{r} \hat{u}^{+}(r)\right)-\frac{1}{\rho_{+}} p^{2} \hat{u}^{+}(r)+\frac{1}{\rho_{+}} r^{2}\left(k^{+}\right)^{2} \hat{u}^{+}(r)=\frac{1}{\rho_{+}} \hat{F}_{1}(r), \quad 0<r<a, \\
& D_{r}\left(\frac{1}{\rho_{-}} D_{r} \hat{u}^{-}(r)\right)-\frac{1}{\rho_{-}} p^{2} \hat{u}^{-}(r)+\frac{1}{\rho_{-}} r^{2}\left(k^{-}\right)^{2} \hat{u}^{-}(r)=\frac{1}{\rho_{-}} \hat{F}_{2}(r), \quad a<r<b, \\
& \hat{u}^{+}(a)-\hat{u}^{-}(a)=0 ; \quad \frac{1}{\rho_{+}} \partial_{r} \hat{u}^{+}(a)-\frac{1}{\rho_{-}} \partial_{r} \hat{u}^{-}(a)=0, \\
& \hat{u}^{+}(0)=0, \text { for } p \neq 0 ; \quad \partial_{r} \hat{u}^{-}(b)-T_{p} \hat{u}^{-}(b)=0,
\end{aligned}
$$

where $\hat{F}_{1}=F_{1}-L_{p}^{+} u^{*+}$ and $\hat{F}_{2}=F_{1}-L_{p}^{-} u^{*-}$. Then denoting

$$
\begin{aligned}
& u(r):= \begin{cases}\hat{u}^{+}(r), & 0<r<a, \\
\hat{u}^{-}(r), & a<r<b,\end{cases} \\
& f(r):= \begin{cases}\hat{F}_{1}(r), & 0<r<a, \\
\hat{F}_{2}(r), & a<r<b,\end{cases} \\
& \rho:= \begin{cases}\rho_{+}, & 0<r<a, \\
\rho_{-}, & a<r<b,\end{cases} \\
& k_{p}(r):= \begin{cases}r^{2}\left(k^{+}\right)^{2}-p^{2}, & 0<r<a, \\
r^{2}\left(k^{-}\right)^{2}-p^{2}, & a<r<b,\end{cases}
\end{aligned}
$$

we can re-state Eqs. (4.6)-(4.9) as

$$
\begin{aligned}
& D_{r}\left(\frac{1}{\rho} D_{r} u(r)\right)+\frac{1}{\rho} k_{p}(r) u(r)=\frac{1}{\rho} f, \quad 0<r<a, \quad a<r<b, \\
& u\left(a^{-}\right)-u\left(a^{+}\right)=0, \quad \frac{1}{\rho_{-}} \partial_{r} u\left(a^{-}\right)-\frac{1}{\rho_{+}} \partial_{r} u\left(a^{+}\right)=0, \\
& u(0)=0 \text { for } p \neq 0, \quad \partial_{r} u(b)-T_{p} u(b)=0 .
\end{aligned}
$$

Now let $P_{N}$ be the polynomial space of degree at most $N$, and define the two-domain spectral-element spaces

$$
\begin{aligned}
& X_{N}:=\left\{u \in C(0, b):\left.u\right|_{(0, a)},\left.u\right|_{(a, b)} \in P_{N}\right\}, \\
& X_{N}^{0}:=\left\{u \in X_{N}: u(0)=0\right\} .
\end{aligned}
$$

The two-domain spectral-element method for Eqs. (4.10)-(4.12) with $p \neq 0$ is then applied to find $u_{N} \in X_{N}^{0}$ such that

$$
\begin{array}{r}
-\left(\frac{1}{\rho} D_{r} u_{N}, \partial_{r}\left(r v_{N}\right)\right)+\frac{1}{\rho_{-}} b^{2} T_{p} u_{N}(b) \bar{v}_{N}(b)+\left(\frac{1}{\rho} k_{p} u_{N}, v_{N}\right)=\left(\frac{1}{\rho} I_{N} f, v_{N}\right), \\
\forall v_{N} \in X_{N}^{0},
\end{array}
$$


where $I_{N}$ is the interpolation operator based on Legendre-Gauss-Lobatto quadratures in $[0, a]$ and $[a, b]$. For $p=0$, we only have to replace $X_{N}^{0}$ by $X_{N}$ in (4.13). The jump in the wave number is located at the interface of the two domains, and it is well-known that the above spectral-element method converges exponentially fast provided $f$ is smooth in each subdomain.

We next construct a set of basis functions for $X_{N}^{0}$, by writing

$$
\begin{cases}x_{1}=2 r / a-1, & 0<r<a, \\ x_{2}=[2 r-(a+b)] /(b-a), & a<r<b .\end{cases}
$$

Thus the basis functions associated with the endpoints of the two-subintervals $(0, a)$ and $(a, b)$ are

$$
\begin{aligned}
& \varphi_{0}(r):= \begin{cases}-\frac{1}{2}\left(x_{1}-1\right), & 0<r<a, \\
0, & a<r<b,\end{cases} \\
& \varphi_{N}(r):= \begin{cases}\frac{1}{2}\left(x_{1}+1\right), & 0<r<a, \\
-\frac{1}{2}\left(x_{2}-1\right), & a<r<b,\end{cases} \\
& \varphi_{2 N}(r):= \begin{cases}0, & 0<r<a, \\
\frac{1}{2}\left(x_{2}+1\right), & a<r<b .\end{cases}
\end{aligned}
$$

If $L_{j}$ denotes the Legendre polynomial of order $j$, we then define the interior basis functions in $[0, a]$ as

$$
\varphi_{j}(r):= \begin{cases}L_{j-1}\left(x_{1}\right)-L_{j+1}\left(x_{1}\right), & 0<r<a, \quad j=1, \cdots, N-1, \\ 0, & a<r<b,\end{cases}
$$

and in $[a, b]$ as

$$
\varphi_{N+j}(r):= \begin{cases}0, & 0<r<a, \\ L_{j-1}\left(x_{2}\right)-L_{j+1}\left(x_{2}\right), & a<r<b, \quad j=1, \cdots, N-1 .\end{cases}
$$

By construction we have

$$
X_{N}^{0}=\operatorname{span}\left\{\varphi_{j}: j=1,2, \cdots, 2 N\right\}
$$


and we set

$$
\begin{array}{ll}
u_{N}=\sum_{i=1}^{2 N} \hat{u}_{i} \varphi_{i}, & \bar{u}=\left(\hat{u}_{1}, \hat{u}_{1}, \cdots, \hat{u}_{2 N}\right)^{T}, \\
A_{k j}=a\left(\varphi_{j}, \varphi_{k}\right), & A=\left(A_{k j}\right)_{1 \leq k, j \leq 2 N}, \\
f_{i}=\left(I_{N} f, \varphi_{i}\right), & \bar{f}=\left(f_{1}, f_{2}, \cdots, f_{2 N}\right)^{T},
\end{array}
$$

such that Eq. (4.13) reduces to the linear system

$$
A \bar{u}=\bar{f} .
$$

(For the case $p=0$, this system needs to be augmented with one more equation.) It is clear that $A$ has two block-diagonal sub-matrices of order $N-1$, and the $N$-th row and column correspond to the interface basis function $\varphi_{N}$. Furthermore, the two sub-matrices are sparse, so the linear system can be solved easily and efficiently by a block-Gaussian elimination process involving $O(N)$ operations.

To summarise, the procedure for solving the problem (2.4)-(2.8) consists of the following steps:

(i) reduce the problem (2.4)-(2.8) to an equivalent problem (2.12)-(2.16) in a bounded domain;

(ii) transform the equivalent problem (2.12)-(2.16) with a general interface $S$ into an equivalent problem (3.2)-(3.6) with a circular interface;

(iii) insert the Taylor expansions (3.7) into (3.2)-(3.6), to derive the sequence of problems (3.10)-(3.14);

(iv) introduce the Fourier expansions for all functions in (3.10)-(3.14), to obtain the sequence of one-dimensional problems (3.15)-(3.19);

(v) solve (3.15)-(3.19) using the spectral-element method, for each $n(0 \leq n \leq M)$ and $p\left(-N_{\theta} \leq p \leq N_{\theta}\right)$; and

(vi) sum up the finite term Taylor expansion (3.7), using a Padé approximation to enlarge the radius of convergence [2] — cf. the Remark 4.1 in Ref. [5] for more detail.

The numerical analysis of the spectral-element method (4.13) used for Eqs. (4.10)-(4.12) can be carried out in a fashion similar to Ref. [22], and the complete error analysis for the transformed field expansion algorithm presented here follows from the general framework established in Ref. [14]. 

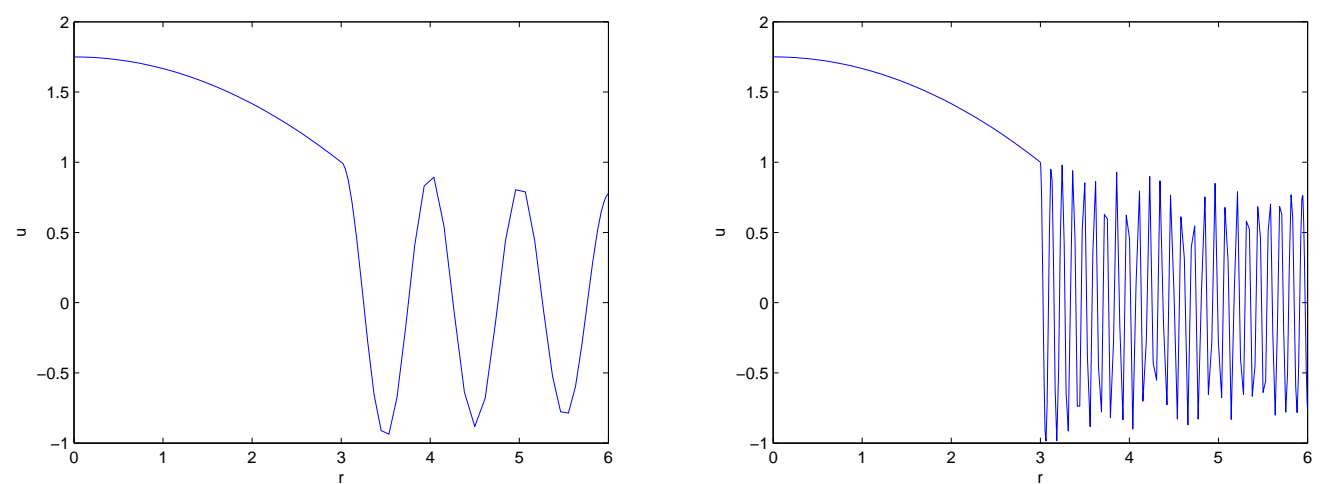

Figure 2: Exact solutions for Example 5.1: left, $\left(k^{+}, k^{-}\right)=(12.5,6.25)$; right, $\left(k^{+}, k^{-}\right)=(102.5,51.25)$.
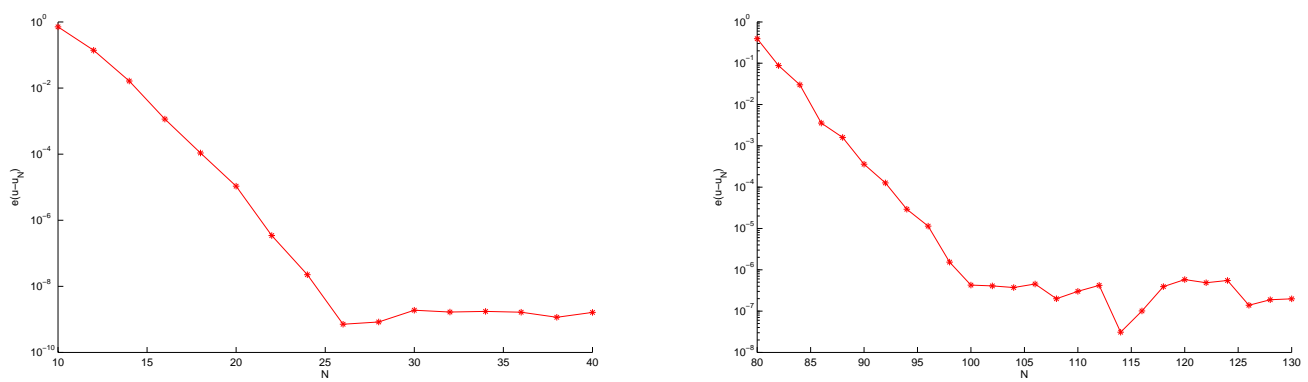

Figure 3: The discrete $L^{2}$ errors for Example 5.1: left, $\left(k^{+}, k^{-}\right)=(12.5,6.25)$; right, $\left(k^{+}, k^{-}\right)=$ $(102.5,51.25)$.

\section{Numerical Results and Discussion}

We now present some numerical experiments to demonstrate the stability and accuracy of our algorithm. We define

$$
\begin{aligned}
E^{2}(u) & =\int_{0}^{b}|u|^{2} d r=\int_{0}^{a}|u|^{2} d r+\int_{a}^{b}|u|^{2} d r \\
& =\frac{1}{2} \int_{-1}^{1}\left|u\left(\frac{1}{2}(t+1)\right)\right|^{2} d t+\frac{b-a}{2} \int_{-1}^{1}\left|u\left(\frac{b-a}{2} t+\frac{a+b}{2}\right)\right|^{2} d t, \\
E_{N}^{2}(u) & =\frac{1}{2} \sum_{i=0}^{N}\left|u\left(\frac{1}{2}\left(t_{i}+1\right)\right)\right|^{2} \omega_{i}+\frac{b-a}{2} \sum_{i=0}^{N}\left|u\left(\frac{b-a}{2} t_{i}+\frac{a+b}{2}\right)\right|^{2} \omega_{i},
\end{aligned}
$$

where $t_{i}$ is Legendre-Gauss-Lobatto point, and we first consider the simple case where the obstacle is a circular domain in order to investigate the numerical convergence by comparing the results with the known exact solution. 
Example 5.1. We fix $a=3, b=6$ and $p=0$, and consider the wave numbers $\left(k^{+}, k^{-}\right)=$ $(12.5,6.25)$ and $\left(k^{+}, k^{-}\right)=(102.5,51.25)$. We set $\rho_{-}=1$ and $\rho_{+}=a$, and note the following exact solution of (4.10)-(4.12):

$$
u(r):= \begin{cases}T_{p}\left(r^{2}-a^{2}\right)+1, & 0<r<a, \\ e^{T_{p}(r-a)}, & a<r<b,\end{cases}
$$

where $T_{p}$ is given in (4.5).

The real parts of the above exact solutions are plotted in Fig. 2, and the discrete $L^{2}$ errors vs. the number of Legendre modes $N$ in each subdomain are plotted in Fig. 3. We observe that the error converges exponentially with $N$ as expected, and that relatively few points are needed to resolve highly oscillatory solutions. However, the error appears to saturate at about $10^{-9}$ for the first case, and $10^{-7}$ for the second case. This is because the problem (4.6)-(4.9) is not positive definite, which leads to relatively large condition numbers for the linear system (4.14), particularly for large wave numbers. We recall that for positive definite problems, for example $k^{ \pm}<0$ in (4.6)-(4.9), the spectral-element method described in the last section would be well-conditioned $[18,20]$.

We now proceed to consider three examples where the obstacles are not circular. In all three we take the incident wave to be $\exp \left(i r k_{-} \cos (\theta)\right), N_{\theta}$ to be the number of Fourier modes in our algorithm, $N_{r}$ the number of Legendre mode in each of the two-subdomains, and $M$ the number of terms in the Taylor expansion. We also write $e_{n, 2}$ for the $L^{2}$ norm of the $n$-th term in the Taylor expansion. We found numerically that $N_{\theta}=35, N_{r}=60$ and $M=35$ are sufficient to obtain at least six-digit accuracy, so we fixed $N_{\theta}=35, N_{r}=60$ and $M=35$.

Example 5.2. We fix $\varepsilon=0.1, a=1, b=2, f(\theta)=\cos (4 \theta)$, and take $\rho_{+} / \rho_{-}=2$ and wave numbers $\left(k^{+}, k^{-}\right)=(16.5,8.25)$.

The shape of the obstacle and the real part of the computed solution are plotted in Fig. 4, and the convergence history of the Taylor expansion is presented in Fig. 5.

Example 5.3. We fix $\varepsilon=0.3, a=2, b=4, f(\theta)=\cos (4 \theta)$, and take $\rho_{+} / \rho_{-}=2$ and wave numbers $\left(k^{+}, k^{-}\right)=(6.5,3.25)$.

The shape of the obstacle and the real part of the computed solution are plotted in Fig. 6, and the convergence history of the Taylor expansion is presented in Fig. 7.

Example 5.4. We fix $\varepsilon=0.6, a=2, b=4, f(\theta)=\cos (4 \theta)$, and take $\rho_{+} / \rho_{-}=2$ and wave numbers $\left(k^{+}, k^{-}\right)=(2.5,1.25)$.

The shape of the obstacle and the real part of the computed solution are plotted in Fig. 8, and the convergence history of the Taylor expansion is presented in Fig. 9.

From these three examples, we observe that the algorithm is quite robust with respect to the size of the perturbation, and that cases with low to moderate wave numbers can be well resolved with relatively few unknowns. 

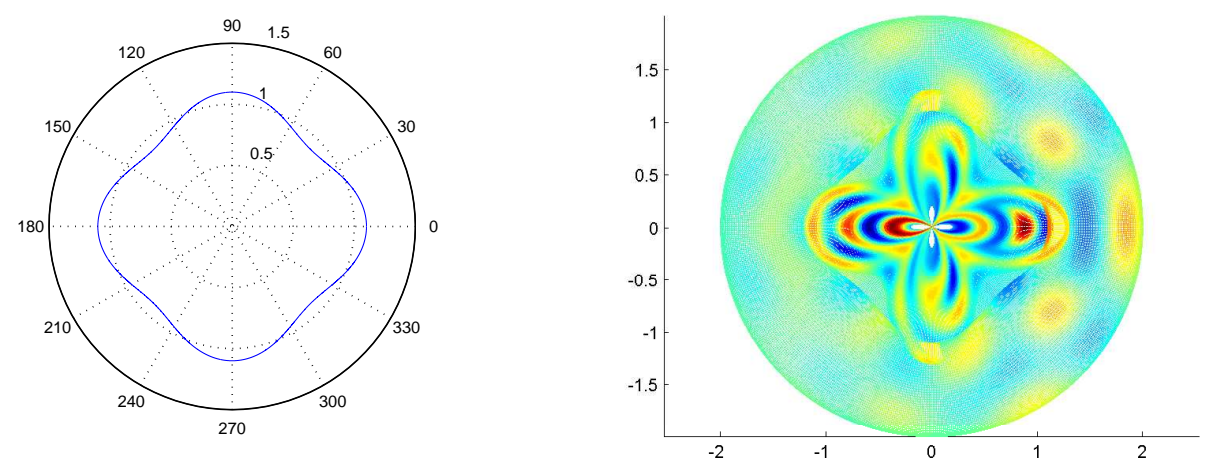

Figure 4: Example 5.2: left, the shape of the obstacle; right, the real part of the numerical solution.

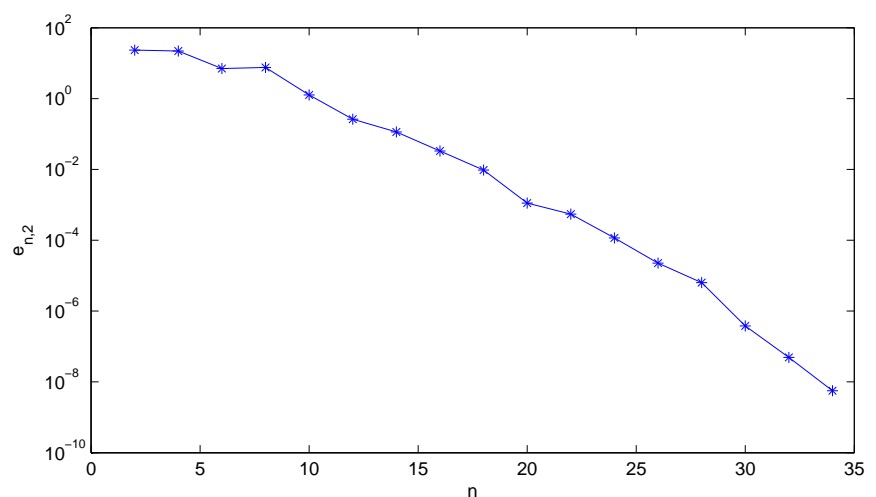

Figure 5: Convergence history of the Taylor expansion for Example 5.2.
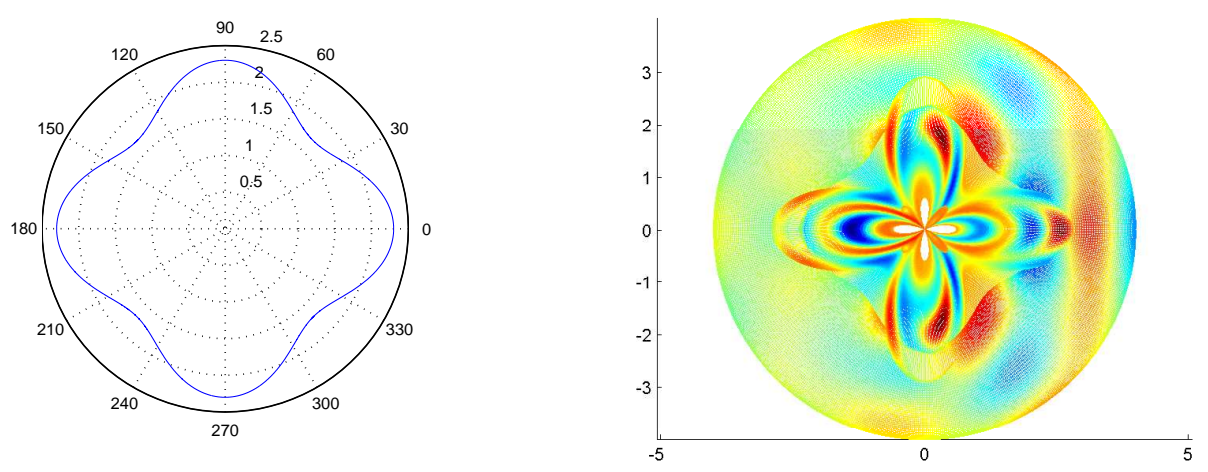

Figure 6: Example 5.3: left, the shape of the obstacle; right, the real part of the numerical solution.

\section{Concluding Remarks}

We have developed an efficient and robust method for solving for the acoustic scattering by an obstacle in an homogeneous compressible fluid. This involves an improved 


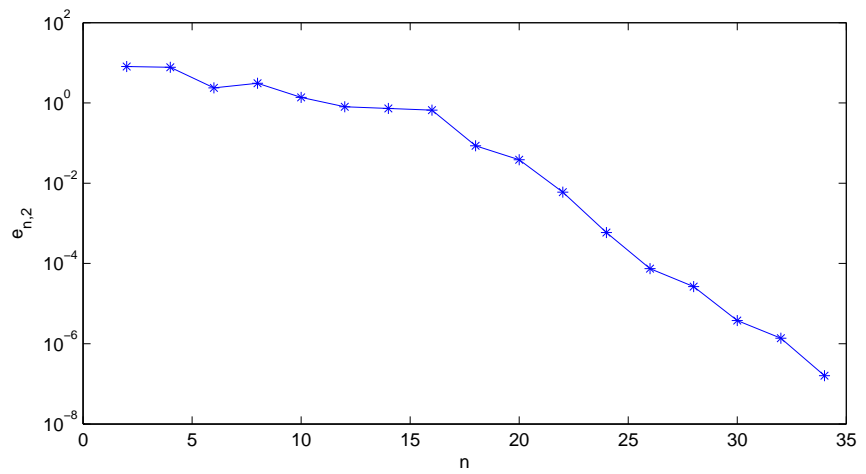

Figure 7: Convergence history of the Taylor expansion for Example 5.3.
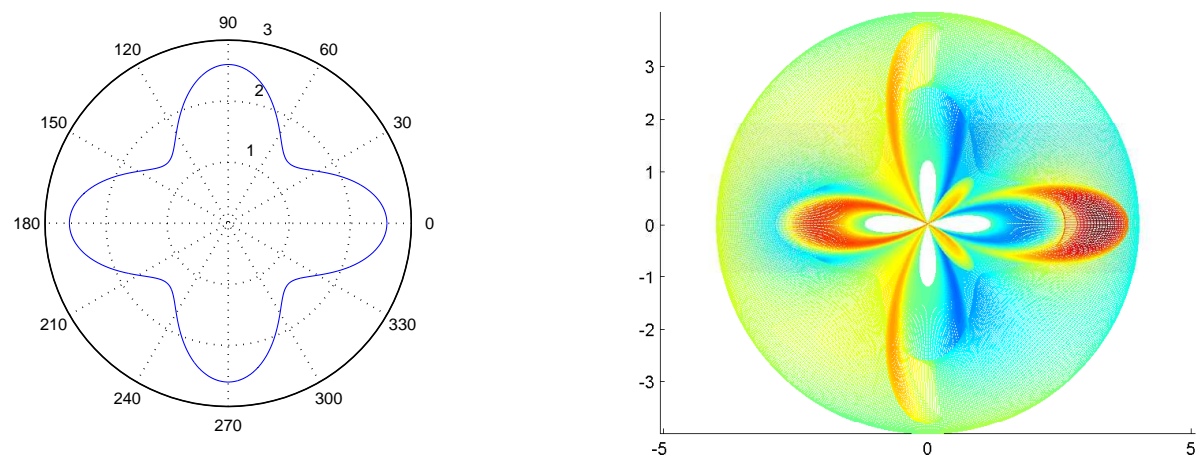

Figure 8: Example 5.4: left, the shape of the obstacle; right, the real part of the numerical solution.

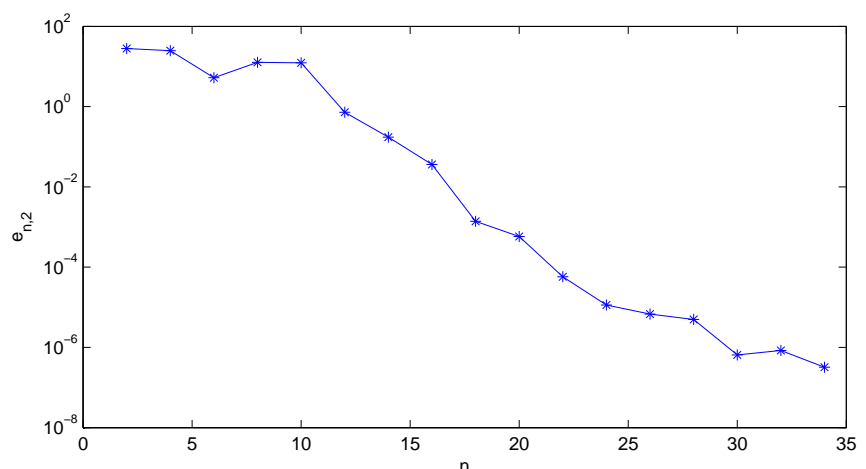

Figure 9: Convergence history of the Taylor expansion for Example 5.4.

boundary perturbation method with a transformed field expansion, coupled with a spectralelement method. The resulting algorithm is semi-direct and consists of a sequence of onedimensional problems, which can be solved by a two-domain spectral-element method. The algorithm is shown to be very efficient and stable for a range of small to moderate 
wave numbers. Although we have only considered two-dimensional obstacles in this article, the method can be extended to three dimensions as in Ref. [4], by using spherical harmonic transforms instead of Fourier transforms.

\section{Acknowledgments}

This work is partially supported by the Chinese NSF grant 91130002 .

\section{A. Detailed Formulae for the Change of Variables and Recursion in Section 3}

With straightforward but tedious calculations, one can obtain the following formulae for $F_{1}$ and $F_{2}$ in Eqs. (3.2)-(3.3):

$$
\begin{aligned}
-a^{2} F_{1}= & 2 a g D_{r^{\prime}}^{2} \tilde{u}^{+}+g^{2} D_{r^{\prime}}^{2} \tilde{u}^{+}+a \partial_{\theta^{\prime}}\left(g \partial_{\theta^{\prime}} \tilde{u}^{+}\right) \\
& -a r^{\prime} \partial_{\theta^{\prime}}\left(\partial_{\theta^{\prime}} g \partial_{r^{\prime}} \tilde{u}^{+}\right)+a g \partial_{\theta^{\prime}}^{2} \tilde{u}^{+}+g \partial_{\theta^{\prime}}\left(g \partial_{\theta^{\prime}} \tilde{u}^{+}\right) \\
& -g r^{\prime} \partial_{\theta^{\prime}}\left(\partial_{\theta^{\prime}} g \partial_{r^{\prime}} \tilde{u}^{+}\right)-a r^{\prime} \partial_{\theta^{\prime}} g \partial_{r^{\prime}} \partial_{\theta^{\prime}} \tilde{u}^{+}-g r^{\prime} \partial_{\theta^{\prime}} g \partial_{r^{\prime}} \partial_{\theta^{\prime}} \tilde{u}^{+} \\
& +\left(\partial_{\theta^{\prime}} g\right)^{2} D_{r^{\prime}}^{2} \tilde{u}^{+}-a \partial_{\theta^{\prime}} g \partial_{\theta^{\prime}} \tilde{u}^{+}-g \partial_{\theta^{\prime}} g \partial_{\theta^{\prime}} \tilde{u}^{+} \\
& +\left(\partial_{\theta^{\prime}} g\right)^{2} D_{r^{\prime}} \tilde{u}^{+}+\sum_{l=1}^{4} c_{l}(g)\left(r^{\prime}\right)^{2}\left(k^{+}\right)^{2} \tilde{u}^{+}
\end{aligned}
$$

where

$$
\begin{array}{ll}
c_{1}(g)=4 a g, & c_{2}(g)=6 g^{2}, \\
c_{3}(g)=\frac{4}{a} g^{3}, & c_{4}(g)=\frac{g^{4}}{a^{2}}
\end{array}
$$

and

$$
\begin{aligned}
-d^{2} F_{2}= & d A \partial_{r^{\prime}} D_{r^{\prime}} \tilde{u}^{-}+d D_{r^{\prime}}\left(A \partial_{r^{\prime}} \tilde{u}^{-}\right)+A \partial_{r^{\prime}}\left(A \partial_{r^{\prime}} \tilde{u}^{-}\right) \\
& -d g \partial_{\theta^{\prime}}^{2} \tilde{u}^{-}-d \partial_{\theta^{\prime}}\left(g \partial_{\theta^{\prime}} \tilde{u}^{-}\right)+g \partial_{\theta^{\prime}}\left(g \partial_{\theta^{\prime}} \tilde{u}^{-}\right)-d B \partial_{r^{\prime}} \partial_{\theta^{\prime}} \tilde{u}^{-} \\
& +B \partial_{r^{\prime}}\left(g \partial_{\theta^{\prime}} \tilde{u}^{-}\right)-d \partial_{\theta^{\prime}}\left(B \partial_{r^{\prime}} \tilde{u}^{-}\right)+g \partial_{\theta^{\prime}}\left(B \partial_{r^{\prime}} \tilde{u}^{-}\right)+B \partial_{r^{\prime}}\left(B \partial_{r^{\prime}} \tilde{u}^{-}\right) \\
& +d\left(\partial_{\theta^{\prime}} g\right) \partial_{\theta^{\prime}} \tilde{u}^{-}-g\left(\partial_{\theta^{\prime}} g\right) \partial_{\theta^{\prime}} \tilde{u}^{-}-\left(\partial_{\theta^{\prime}} g\right) B \partial_{r^{\prime}} \tilde{u}^{-}+\sum_{j=1}^{4} \tilde{c}_{j}(g)\left(k^{-}\right)^{2} \tilde{u}^{-}
\end{aligned}
$$

where

$$
\begin{aligned}
& \tilde{c}_{1}(g)=-2 d g\left(r^{\prime}\right)^{2}+2 d A r^{\prime}, \tilde{c}_{2}(g)=g^{2}\left(r^{\prime}\right)^{2}-4 g A r^{\prime}+A^{2}, \\
& \tilde{c}_{3}(g)=\frac{2}{d} A g^{2} r^{\prime}-\frac{2}{d} g A^{2}, \tilde{c}_{4}(g)=\frac{1}{d} g^{2} A^{2} .
\end{aligned}
$$


Similarly, we can derive the following formulae for $F_{1 n}, F_{2 n}, Q_{n}$ and $J_{n}$ in Eqs. (3.10)(3.14):

$$
\begin{aligned}
-a^{2} F_{1 n}= & 2 a f D_{r^{\prime}}^{2} \tilde{u}_{n-1}^{+}+f^{2} D_{r^{\prime}}^{2} \tilde{u}_{n-2}^{+}+a \partial_{\theta^{\prime}}\left(f \partial_{\theta^{\prime}} \tilde{u}_{n-1}^{+}\right) \\
& -a r^{\prime} \partial_{\theta^{\prime}}\left(\partial_{\theta^{\prime}} f \partial_{r^{\prime}} \tilde{u}_{n-1}^{+}\right)+a f \partial_{\theta^{\prime}}^{2} \tilde{u}_{n-1}^{+}+f \partial_{\theta^{\prime}}\left(f \partial_{\theta^{\prime}} \tilde{u}_{n-2}^{+}\right) \\
& -f r^{\prime} \partial_{\theta^{\prime}}\left(\partial_{\theta^{\prime}} f \partial_{r^{\prime}} \tilde{u}_{n-2}^{+}\right)-a r^{\prime} \partial_{\theta^{\prime}} f \partial_{r^{\prime}} \partial_{\theta^{\prime}} \tilde{u}_{n-1}^{+}-f r^{\prime} \partial_{\theta^{\prime}} f \partial_{r^{\prime}} \partial_{\theta^{\prime}} \tilde{u}_{n-2}^{+} \\
& +\left(\partial_{\theta^{\prime}} f\right)^{2} D_{r^{\prime}}^{2} \tilde{u}_{n-2}^{+}-a \partial_{\theta^{\prime}} f \partial_{\theta^{\prime}} \tilde{u}_{n-1}^{+}-f \partial_{\theta^{\prime}} f \partial_{\theta^{\prime}} \tilde{u}_{n-2}^{+} \\
& +\left(\partial_{\theta^{\prime}} f\right)^{2} D_{r^{\prime}} \tilde{u}_{n-2}^{+}+\sum_{l=1}^{4} c_{l}(f)\left(r^{\prime}\right)^{2}\left(k^{+}\right)^{2} \tilde{u}_{n-l}^{+},
\end{aligned}
$$

and

$$
\begin{aligned}
-d^{2} F_{2 n}= & d \tilde{A} \partial_{r^{\prime}} D_{r^{\prime}} \tilde{u}_{n-1}^{-}+d D_{r^{\prime}}\left(\tilde{A} \partial_{r^{\prime}} \tilde{u}_{n-1}^{-}\right)+\tilde{A} \partial_{r^{\prime}}\left(\tilde{A} \partial_{r^{\prime}} \tilde{u}_{n-2}^{-}\right) \\
& -d f \partial_{\theta^{\prime}}^{2} \tilde{u}_{n-1}^{-}-d \partial_{\theta^{\prime}}\left(f \partial_{\theta^{\prime}} \tilde{u}_{n-1}^{-}\right)+f \partial_{\theta^{\prime}}\left(f \partial_{\theta^{\prime}} \tilde{u}_{n-2}^{-}\right)-d \tilde{B} \partial_{r^{\prime}} \partial_{\theta^{\prime}} \tilde{u}_{n-1}^{-} \\
& +\tilde{B} \partial_{r^{\prime}}\left(f \partial_{\theta^{\prime}} \tilde{u}_{n-2}^{-}\right)-d \partial_{\theta^{\prime}}\left(\tilde{B} \partial_{r^{\prime}} \tilde{u}_{n-1}^{-}\right)+f \partial_{\theta^{\prime}}\left(\tilde{B} \partial_{r^{\prime}} \tilde{u}_{n-2}^{-}\right)+\tilde{B} \partial_{r^{\prime}}\left(\tilde{B} \partial_{r^{\prime}} \tilde{u}_{n-2}^{-}\right) \\
& +d\left(\partial_{\theta^{\prime}} f\right) \partial_{\theta^{\prime}} \tilde{u}_{n-1}^{-}-f\left(\partial_{\theta^{\prime}} f\right) \partial_{\theta^{\prime}} \tilde{u}_{n-2}^{-}-\left(\partial_{\theta^{\prime}} f\right) \tilde{B} \partial_{r^{\prime}} \tilde{u}_{n-2}^{-}+\sum_{j=1}^{4} \tilde{d}_{j}(f)\left(k^{-}\right)^{2} \tilde{u}_{n-j}^{-},
\end{aligned}
$$

where

$$
\begin{gathered}
\tilde{A}=f\left(b-r^{\prime}\right), \quad \tilde{B}=\left(\partial_{\theta^{\prime}} f\right)\left(b-r^{\prime}\right) . \\
\tilde{d}_{1}(f)=-2 d f\left(r^{\prime}\right)^{2}+2 d \tilde{A} r^{\prime}, \quad \tilde{d}_{2}(f)=f^{2}\left(r^{\prime}\right)^{2} ! 4 f \tilde{A} r^{\prime}+\tilde{A}^{2}, \\
\tilde{d}_{3}(f)=\frac{2}{d} \tilde{A} f^{2} r^{\prime}-\frac{2}{d} f \tilde{A}^{2}, \quad \tilde{d}_{4}(f)=\frac{1}{d} f^{2} \tilde{A}^{2} ; \\
-a^{3} d Q_{n}=\left(2 a^{2} d f-a^{3} f\right) \frac{\partial \tilde{u}_{n-1}^{+}}{\partial r^{\prime}}+\left(a d f^{2}-2 a^{2} f^{2}+a d\left(\partial_{\theta^{\prime}} f\right)^{2}\right) \frac{\partial \tilde{u}_{n-2}^{+}}{\partial r^{\prime}} \\
-\left(a f^{3}+a f\left(\partial_{\theta^{\prime}} f\right)^{2}\right) \frac{\partial \tilde{u}_{n-3}^{+}}{\partial r^{\prime}}-a d \partial_{\theta^{\prime}} f \frac{\partial \tilde{u}_{n-1}^{+}}{\partial \theta^{\prime}}+\left(a f \partial_{\theta^{\prime}} f-d f \partial_{\theta^{\prime}} f\right) \frac{\partial \tilde{u}_{n-2}^{+}}{\partial \theta^{\prime}} \\
+f^{2} \partial_{\theta^{\prime}} f \frac{\partial \tilde{u}_{n-3}^{+}}{\partial \theta^{\prime}}-\delta\left\{3 a^{2} d f \frac{\partial \tilde{u}_{n-1}^{-}}{\partial r^{\prime}}+\left(3 a d f^{2}-a^{2}\left(\partial_{\theta^{\prime}} f\right)^{2}+a b\left(\partial_{\theta^{\prime}} f\right)^{2}\right) \frac{\partial \tilde{u}_{n-2}^{-}}{\partial r^{\prime}}\right. \\
+\left(d f^{3}-a f\left(\partial_{\theta^{\prime}} f\right)^{2}+b f\left(\partial_{\theta^{\prime}} f\right)^{2}\right) \frac{\partial \tilde{u}_{n-3}^{-}}{\partial r^{\prime}}-a d \partial_{\theta^{\prime}} f \frac{\partial \tilde{u}_{n-1}^{-}}{\partial \theta^{\prime}}+\left(a f \partial_{\theta^{\prime}} f-d f \partial_{\theta^{\prime}} f\right) \frac{\partial \tilde{u}_{n-2}^{-}}{\partial \theta^{\prime}} \\
+f^{2} \partial_{\theta^{\prime}} f \frac{\partial \tilde{u}_{n-3}^{-}}{\partial \theta^{\prime}}+a^{3} d i p\left(\theta^{\prime}\right)\left(\tilde{\phi}_{n}-\frac{f}{d} \tilde{\phi}_{n-1}\right)+3 a^{2} d f i p\left(\theta^{\prime}\right)\left(\tilde{\phi}_{n-1}-\frac{f}{d} \tilde{\phi}_{n-2}\right)
\end{gathered}
$$




$$
\begin{aligned}
& +\left(3 a d f^{2}-a^{2}\left(\partial_{\theta^{\prime}} f\right)^{2}+a b\left(\partial_{\theta^{\prime}} f\right)^{2}\right) i p\left(\theta^{\prime}\right)\left(\tilde{\phi}_{n-2}-\frac{f}{d} \tilde{\phi}_{n-3}\right) \\
& +\left(d f^{3}-a f\left(\partial_{\theta^{\prime}} f\right)^{2}+b f\left(\partial_{\theta^{\prime}} f\right)^{2}\right) i p\left(\theta^{\prime}\right)\left(\tilde{\phi}_{n-3}-\frac{f}{d} \tilde{\phi}_{n-4}\right) \\
& -a d i \partial_{\theta^{\prime}} f\left(a \partial_{\theta^{\prime}} p\left(\theta^{\prime}\right) \tilde{\phi}_{n-1}+\left(\partial_{\theta^{\prime}} f p\left(\theta^{\prime}\right)+f \partial_{\theta^{\prime}} p\left(\theta^{\prime}\right)\right) \tilde{\phi}_{n-2}\right) \\
& +i\left(a f \partial_{\theta^{\prime}} f-d f \partial_{\theta^{\prime}} f\right)\left(a \partial_{\theta^{\prime}} p\left(\theta^{\prime}\right) \tilde{\phi}_{n-2}+\left(\partial_{\theta^{\prime}} f p\left(\theta^{\prime}\right)+f \partial_{\theta^{\prime}} p\left(\theta^{\prime}\right)\right) \tilde{\phi}_{n-3}\right) \\
& \left.+i f^{2} \partial_{\theta^{\prime}} f\left(a \partial_{\theta^{\prime}} p\left(\theta^{\prime}\right) \tilde{\phi}_{n-3}+\left(\partial_{\theta^{\prime}} f p\left(\theta^{\prime}\right)+f \partial_{\theta^{\prime}} p\left(\theta^{\prime}\right)\right) \tilde{\phi}_{n-4}\right)\right\}
\end{aligned}
$$

and

$$
d J_{n}=-f T \tilde{u}_{n-1}^{-}\left(b, \theta^{\prime}\right)
$$

\section{References}

[1] P.G. Bergmann, The wave equation in a medium with a variable index of refraction, J. Acoust. Soc. Am. 17, 329-333 (1946).

[2] O.P. Bruno and F. Reitich, Solution of a boundary value problem for the Helmholtz equation via variation of the boundary into the complex domain, Proc. Roy. Soc. Edinburgh A - Mathematics 122, 317-340 (1992).

[3] D.L. Colton and R. Kress, Inverse Acoustic and Electromagnetic Scattering Theory, Springer Verlag (1998).

[4] Q. Fang, D.P. Nicholls and J. Shen, A stable, high-order method for three-dimensional, boundedobstacle, acoustic scattering. J. Comput. Phys. 224, 1145-1169 (2007).

[5] Y. He, D.P. Nicholls and J. Shen, An efficient and stable spectral method for electromagnetic scattering from a layered periodic structure, J. Comput. Phys. 231, 3007-3022 (2012).

[6] P.A. Martin, Acoustic scattering by inhomogeneous spheres, J. Acoust. Soc. Am. 111, 2013-2018 (2002).

[7] D.P. Nicholls and N. Nigam, Exact non-reflecting boundary conditions on general domains, J. Comput. Phys. 194, 278-303 (2004).

[8] D.P. Nicholls and F. Reitich, A new approach to analyticity of Dirichlet-Neumann operators, Proc. Roy. Soc. Edinburgh A - Mathematics 131, 1411-1434 (2001).

[9] D.P. Nicholls and F. Reitich, Stability of high-order perturbative methods for the computation of Dirichlet-Neumann operators, J. Comput. Phys. 170, 276-298 (2001).

[10] D.P. Nicholls and F. Reitich, Analytic continuation of Dirichlet-Neumann operators, Numer. Math. 94, 107-146 (2003).

[11] D.P. Nicholls and F. Reitich, Shape deformations in rough-surface scattering: Cancellations, conditioning, and convergence, JOSA A 21, 590-605 (2004).

[12] D.P. Nicholls and F. Reitich, Shape deformations in rough-surface scattering: Improved algorithms, JOSA A 21, 606-621 (2004).

[13] D.P. Nicholls and J. Shen, A stable high-order method for two-dimensional bounded-obstacle scattering, SIAM J. Sci. Comput. 28, 1398-1419 (2006).

[14] D.P. Nicholls and J. Shen, A rigorous numerical analysis of the transformed field expansion method, SIAM J. Numer. Anal. 47, 2708-2723 (2009).

[15] Lord Rayleigh, On the dynamical theory of gratings, Soc. London Ser. A 79, 399-416 (1907).

[16] F. Reitich and K.K. Tamma, State-of-the-art, trends, and directions in computational electromagnetics, Comput. Model. Engr. Sci. 5, 287-294 (2004). 
[17] S.O. Rice, Reflection of electromagnetic waves from slightly rough surfaces, Comm. Pure Appl. Math. 4, 351-378 (1951).

[18] J. Shen, Efficient Spectral-Galerkin method I. Direct solvers of second-and fourth-order equations using Legendre polynomials, SIAM J. Sci. Comput. 15, 1489-1508 (1994).

[19] J. Shen, Efficient Spectral-Galerkin method II. Direct solvers of second-and fourth-order equations using Chebyshev polynomials, SIAM J. Sci. Comput. 16, 74-87 (1995).

[20] J. Shen, Efficient Spectral-Galerkin methods III: Polar and cylindrical geometries, SIAM J. Sci. Comput. 18, 1583-1604 (1997).

[21] J. Shen, Efficient Spectral-Galerkin methods IV. Spherical geometries, SIAM J. Sci. Comput. 20, 1438-1455 (1999).

[22] J. Shen and L.-L. Wang, Analysis of a Spectral-Galerkin approximation to the Helmholtz equation in exterior domains, SIAM J. Numer. Anal. 45, 1954-1978 (2007).

[23] K.F. Warnick and W.C. Chew, Numerical simulation methods for rough surface scattering, Waves in Random Media 11, 1-30 (2001). 